\title{
Persistent Effects of Adapalene Gel After Chemical Peeling with Glycolic Acid in Patients with Acne Vulgaris
}

\author{
Mikiko Uede $^{*}, 1$, Chikako Kaminaka ${ }^{1,2}$, Nozomi Yonei ${ }^{3}$, Fukumi Furukawa ${ }^{1}$ and Yuki Yamamoto ${ }^{1,2}$ \\ ${ }^{I}$ Department of Dermatology, Wakayama Medical University, Japan \\ ${ }^{2}$ Department of Cosmetic Dermatology and Photomedicine, Wakayama Medical University, Japan \\ ${ }^{3}$ Department of Dermatology, Public Naga Hospital, Japan
}

\begin{abstract}
We investigated the usefulness of adapalene gel as maintenance therapy following chemical peeling with glycolic acid in patients with acne vulgaris. The study period was 14 weeks. The subjects were 23 patients with mild to moderate acne vulgaris (1 male, 22 females). After chemical peeling (CP) of the face was performed 3 times at 2-week intervals, adapalene was applied for 6 weeks using a randomized, double-blind half-side method. On the day of observation, dermatologists examined dermal findings, and measurement was conducted using instruments to analyze the physiological skin function. After the third session of $\mathrm{CP}$ was completed, both the inflammatory and non-inflammatory lesion counts significantly decreased. Subsequently, on the adapalene-treated side there were no change in the inflammatory and non-inflammatory lesion counts after the CP 3 times, but on placebo-treated side, there significant increase in the inflammatory and non inflammatory lesion counts. Concerning the results of measurement with instruments, the sebum capacity significantly decreased after the third session of CP. Subsequently, there were no changes after the 6-week application of adapalene or a placebo. These results suggest that post-CP adapalene application is an effective acne treatment method to improve efficacy and treatment adherence.
\end{abstract}

Keywords: Acne vulgaris(AV), chemical peeling(CP), adapalene.

\section{INTRODUCTION}

Acne vulgaris is frequent in clinical practice. It is a chronic, inflammatory dermal disease involving sebaceous hair follicles [1]. Its clinical characteristics include seborrhea, comedones, red papules, and pustules [1]. This disease frequently develops on the faces and thoracic/dorsal regions of persons aged 10 to 29 years, and is experienced by more than $90 \%$ of Japanese persons $[2,3]$.

Chemical peeling $(\mathrm{CP})$ is a technique to treat dermal symptoms related to acne, pigment anomalies, and photoaging or cosmetically improve the skin (anti-aging, reduction of spots/dullness, and improvement in the skin quality) [4]. In patients with acne, the thickening cornified layer of the hair follicle infundibulais exfoliated, reducing sebum retention in hair follicles. In particular, this procedure is effective for pimples [4]. Glycolic acid belongs to $\alpha$ hydroxy acids, with the smallest molecular weight. It is appropriate for peeling of the most superficial (level 1) and superficial (level 1,2) layers [5]. Neither systemic toxicity nor serious complications have been reported, and various products are commercially available. In 2008, the Japanese Dermatological Association prepared the "Guidelines for Acne Vulgaris Treatment" for Japanese [6]. Concerning inflammatory/non-inflammatory lesion, glycolic and salicylic

*Address correspondence to this author at the Department of Dermatology, Wakayama Medical University, Postal code: 641-0012, 811-1, Kimiidera, Wakayama City, Wakayama Prefecture, Japan; Tel.: 073-441-0661;

Fax: 073-441-1908; E-mail: kifubi@wakayama-med.ac.jp acids (macrogol base) were recommended as recommendation grade $\mathrm{C} 1$ (recommended as options, although there was little evidence) [5-7]. On the other hand, adapalene (6-[3-(1adamantyl)-4-methoxyphenyl]-2-naphthoic acid), a naphthoic acid derivative [8], was approved as an external retinoid preparation in Japan in 2008 [9]. In patients with acne, this drug corrects the alteration of the hyperkeratinization process of the hair follicle infundibular, reducing microcomedones, comedones and inflammatory lesions. Furthermore, it was also strongly recommended as maintenance therapy after the reduction of inflammation. In the guidelines, the recommendation grade was established as A (Strongly recommended to perform (there should be at least one level I or II study that supports effectiveness) [5-7]. However, no study has compared CP with adapalene, although CP may exhibit immediate actions on comedones and cosmetic effects such as whitening [4]. In this study, we used an external adapalene preparation (recommendation grade A) after $\mathrm{CP}$, which reduces lesion in the early phase, and examined its usefulness for acne vulgaris treatment.

\section{SUBJECTS AND METHODS}

\section{Subjects}

The subjects were 23 patients with mild to moderate acne, aged over 20 years, in whom there was no laterality in the acne count, and from whom written informed consent was obtained in the Department of Dermatology, Wakayama Medical University between 2009 and 2010. Patients were excluded if they had cyst, scar, and keloids, or other dematologic conditions requiring systemic treatment. 
Exclusion criteria included males/females aged 19 years or younger and pregnant or lactating women. The purpose and contents of this study were examined and approved by the Ethic Review Board of Wakayama Medical University in accordance with the Helsinki Declaration.

\section{Methods}

The study period was 12 weeks. Chemical peeling with $40 \%$ glycolic acid (pH3.2) was performed 3 times at 2-week intervals [9]. From 2 weeks after the completion of CP, adapalene or a placebo (supplied by Galderma, Inc.) was applied to the half-face before bedtime every day for 6 weeks. Each drug was randomly assigned using the doubleblind method.

\section{EVALUATION}

The severity of acne was evaluated according to the guidelines [6]. The exanthema count and skin condition were examined by dermatologists every two weeks (total: 7 times). For physiological skin function analysis, the water content $(\%)$ was measured using a CORNEOMETER ${ }^{\circledR}$, the sebum capacity $\left(\mu \mathrm{g} / \mathrm{cm}^{2}\right)$ using a SEBMETER $\AA$, and the transepidermal water loss (TEWL) $\left(\mathrm{g} / \mathrm{cm}^{2}\right)$ using a TEWAMETER ${ }^{\circledR}$ (Multi-probe adaptor, Courage + Khazaka Co., Ltd., Cologne, Germany) at the start of this study, 2 weeks after the completion of the third session of CP, and 6 weeks after the start of adapalene application (total: 3 times) in a room with constant temperature $\left(22 \sim 23^{\circ} \mathrm{C}\right)$ and humidity (relative humidity $40 \sim 45 \%$ ).

\section{STATISTICAL ANALYSIS}

Statistical analysis was conducted using Wilcoxon's ttest. A p-value $<0.05$ was regarded as significant.

\section{RESULTS}

The subjects were 23 patients with acne vulgaris ( 1 male, 22 females), with a mean age of $25.3 \pm 5.8$ (standard deviation) years.

\section{Changes in the Lesion}

Time dependent changes in the inflammatory lesion are shown in Fig. (1). After the completion of the third session of $\mathrm{CP}\left(6^{\text {th }}\right.$ week $)$, the lesion significantly decreased in comparison with the pre-study value $(\mathrm{p}<0.001)$. Subsequently, there was a significant decrease after the 6week $\left(12^{\text {th }}\right.$ week) application of adapalene in comparison with the pre-study value $(p<0.01)$. However, 6 weeks $\left(12^{\text {th }}\right.$ week) after the start of application in adapalene group, there was no significant difference in comparison with the value after the third session of CP ( $6^{\text {th }}$ week), although there was a slight decrease from 2 weeks after the start of application $\left(8^{\text {th }}\right.$ week). In the placebo group, the lesion significantly increased in comparison with that after the third session of CP $\left(12^{\text {th }}\right.$ week $)(\mathrm{p}<0.01)$.

The changes in the non-inflammatory lesion are shown in Fig. (2). After the third session of CP ( $6^{\text {th }}$ week $)$, the noninflammatory lesion significantly decreased in comparison with the pre-study value $(\mathrm{p}<0.001)$. Subsequently, there were significant decreases after the 6-week ( $12^{\text {th }}$ week) application of adapalene or the placebo in comparison with the pre-study value $(p<0.01)$. In the adapalene group, there was no significant difference in comparison with the value after the third session of CP ( $6^{\text {th }}$ week), although there was a slight decrease. In the placebo group, the exanthema was significantly greater than after the third session of $C P\left(6^{\text {th }}\right.$ week) $(\mathrm{p}<0.05)$.

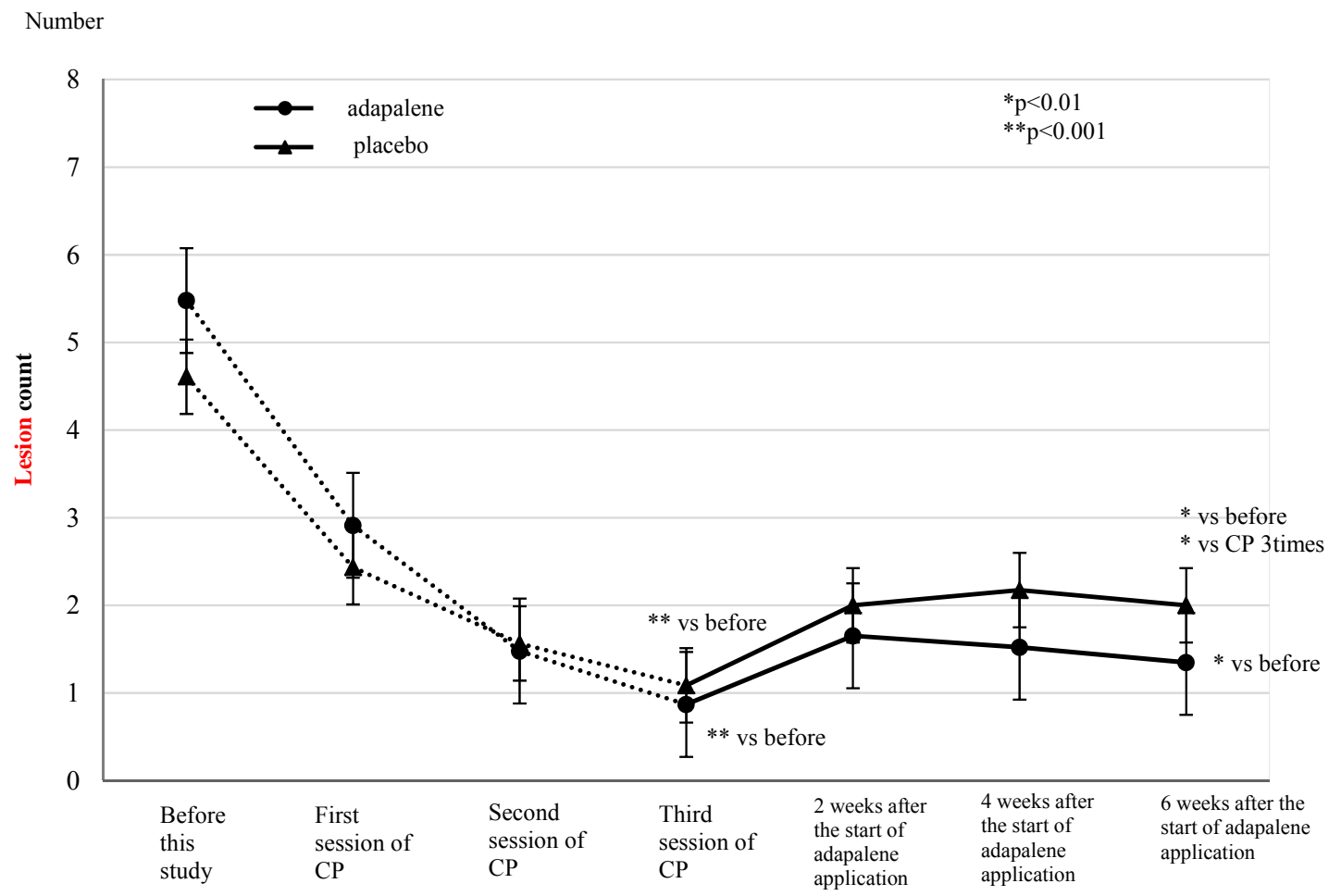

Fig. (1). Changes in the inflammatory lesion count $(\mathrm{N}=23)$. 


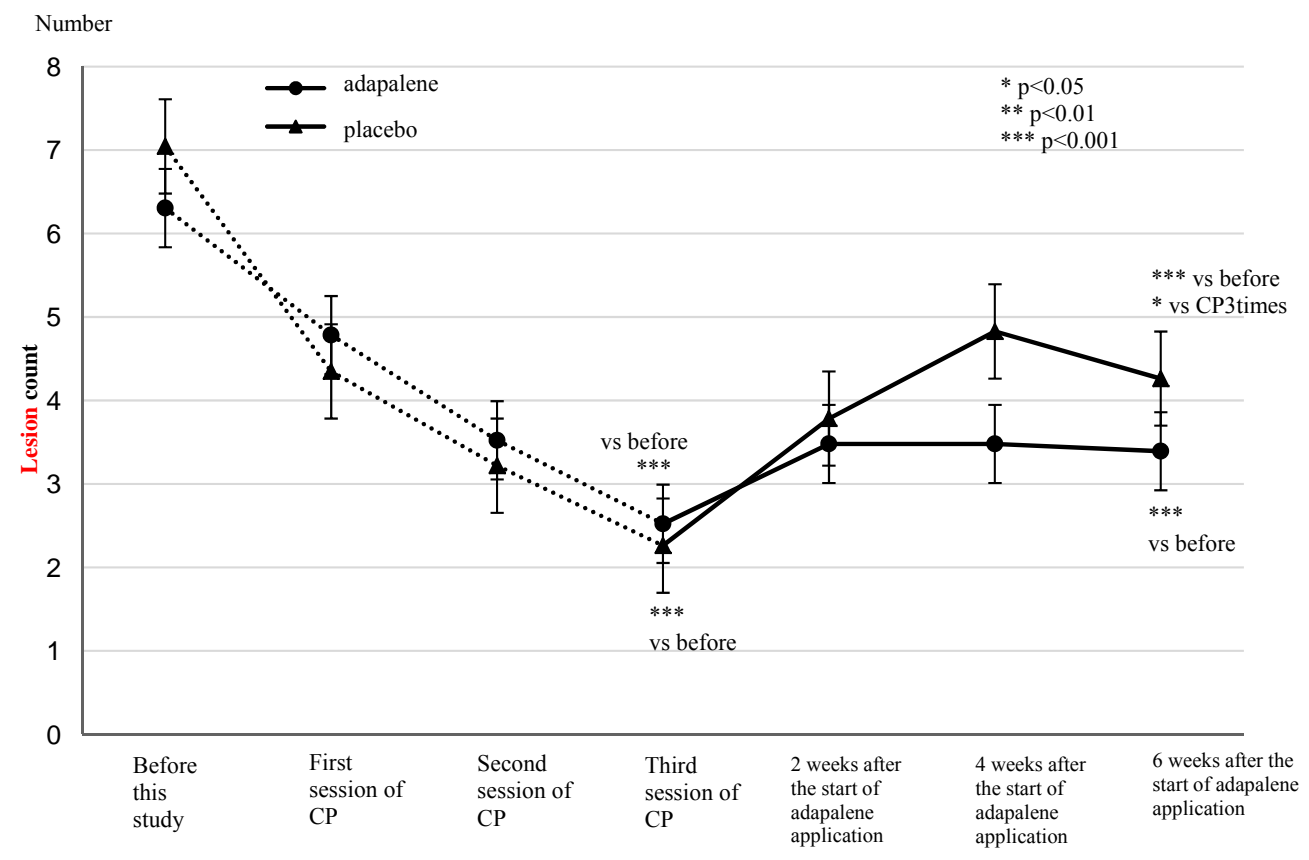

Fig. (2). Changes in the non-inflammatory lesion count $(\mathrm{N}=23)$.

\section{Analysis of the Physiological Skin Function}

\section{Changes in the Sebum Secretion}

After the third session of $\mathrm{CP}$, the sebum secretion significantly decreased in comparison with the pre-study value $(p<0.05)$. Subsequently, there were slight increases in both the adapalene and placebo groups, although there were no significant differences (Fig. 3).

\section{Changes in TEWL}

After the third session of $\mathrm{CP}$, the TEWL value significantly decreased in comparison with the pre-study value $(\mathrm{p}<0.05)$. Subsequently, the values in the adapalene and placebo groups were significantly higher than after the third session of CP (Fig. 4).

\section{Changes in the Cornified Layer Water Content}

There was no significant difference in the cornified layer water content before and after this study.

\section{Safety}

During the study period, there were no side effects of CP. In the adapalene group, more than $90 \%(21 / 23)$ of the patients complained of dryness, desquamation, erythema, or

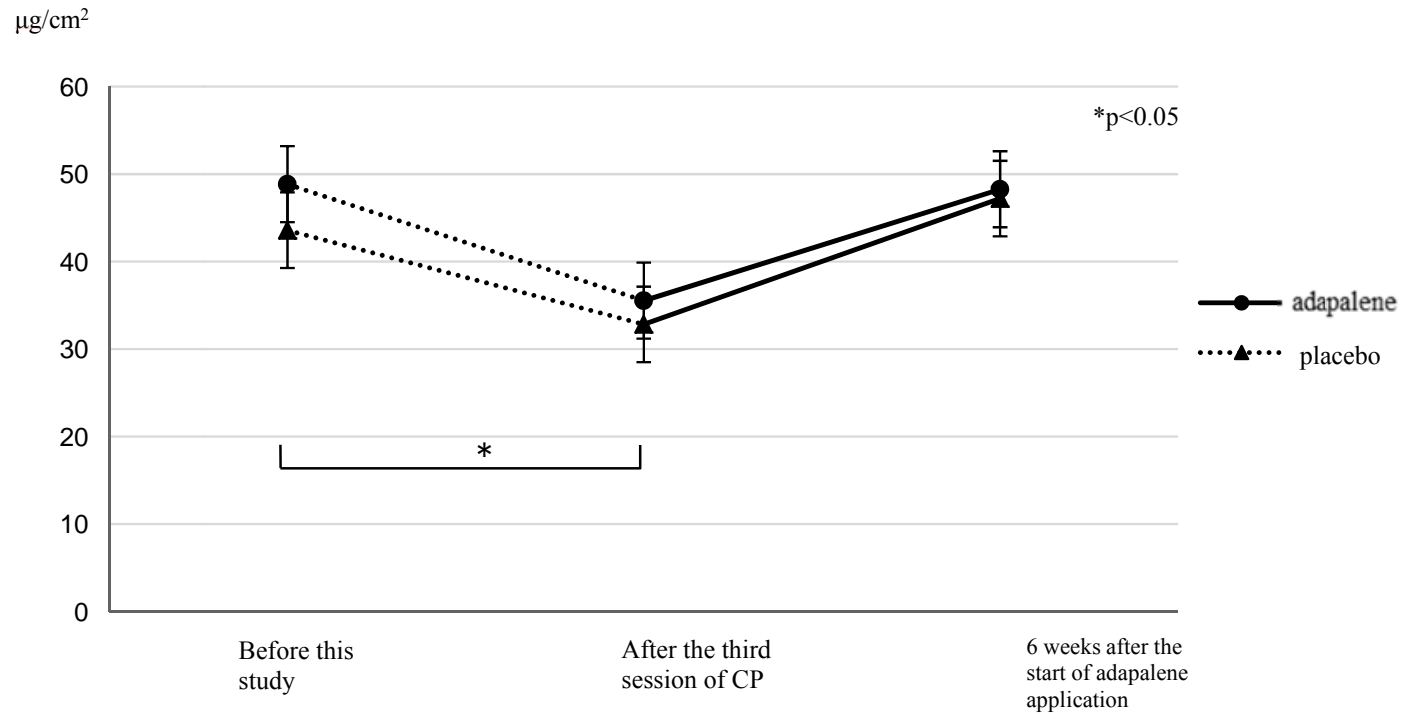

Fig. (3). Changes in the sebum secretion $(\mathrm{N}=23)$. 


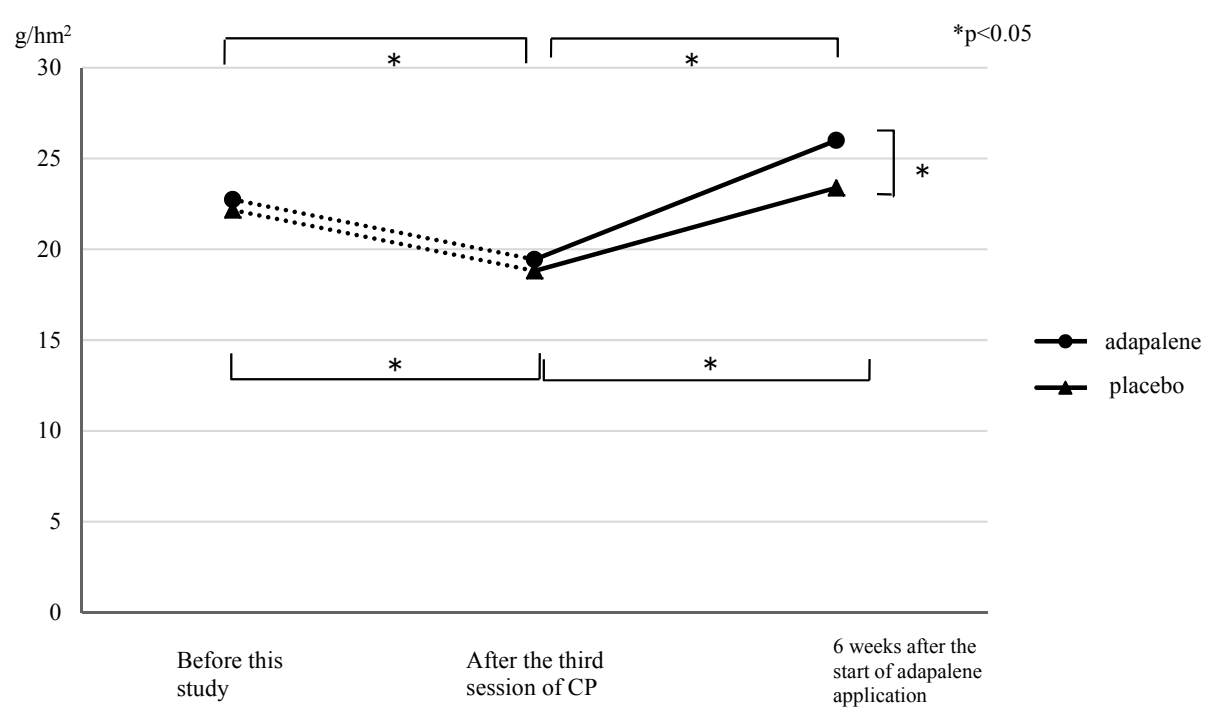

Fig. (4). Changes in the TEWL $(\mathrm{N}=23)$.

irritation within 2 weeks. However, in all patients, it was possible to continue treatment until the completion of this study.

\section{DISCUSSION}

Recently, patients have been strongly interested in acne treatment, and acne has been reviewed as an important dermal disease. Chemical peeling shows immediate actions on acne vulgaris, and its results are satisfactory for females and young patients, who have a strong esthetic sense. According to several studies, $\mathrm{CP}$ with glycolic acid significantly decreases the lesion count 2 weeks after the start of treatment; glycolic acid may exhibit more immediate actions compared to adapalene [10,11]. However, as a limitation of $\mathrm{CP}$, continuous visits are required, which is stressful. In this study, we examined the usefulness of adapalene application as maintenance therapy following $\mathrm{CP}$ with glycolic acid. After the third session of $\mathrm{CP}$, both inflammatory and non-inflammatory lesion significantly reduced. Subsequently, adapalene was applied for 6 weeks. The lesion count slightly increased with no statistical significance 2 to 4 weeks after the start of application, but there was a significant decrease in comparison with the prestudy value. These results suggest that adapalene application is useful for maintaining the therapeutic effects of CP.

Acne is often thought to affect the teenaged group. However, a significant number of patients either continue to experience acne or develop new-onset acne after the teenaged years. As acne frequently develops on the face, it influences the patient's quality of life (QOL) [12]. In particular, QOL of most patients with post adolescent acne markedly reduces because of their strong esthetic sense $[12,13]$.

In this study, the mean age of subjects are 25.3 \pm 5.8 (standard deviation) years, mostof who have post-adlescent acne [14]. Irregular menstruation, mental stress, and hormones, especially androgen, are closely involved in postadlescent acne [15]. Androgen acts on the sebaceous gland, promoting sebum production and leading to comedones formation through the excessive cornification of hair follicles. Furthermore, marked mental stress enhances the production of corticotropin-releasing and adrenocorticotropic hormones, increasing the secretion of androgen. As a result, acne exacerbates, reducing the treatment responsiveness [15].

For physiological skin function analysis, the sebum secretion, TEWL, and cornified layer water content were measured before this study, after the third session of CP, and after the completion of this study. The sebum secretion significantly decreased after the third session of $\mathrm{CP}$. Subsequently, it increased after the application of adapalene/a placebo. As the facial secretion of sebum in patients with acne is higher than in healthy adults, sebum secretion is associated with the onset of acne [16]. Several studies have reported that retinoid/hormonal/laser therapies decrease sebum secretion through the destruction/reduction of the sebaceous gland, leading to the remission of acne. However, there are few studies on a CP-related decrease in sebum secretion [17]. A study indicated that glycolic acid penetratedpores of the skin, acting on the sebaceous gland cells and contributing to a decrease in sebum secretion [17]. There was a significant decrease in the TEWL after CP, whereas there was a significant increase following adapalene application. This was possibly because the side effects of adapalene, such as erythema, desquamation, and dryness, reduced the barrier function of the skin $[9,18]$. There were no changes in the water content before and after this study.

The results of this study suggest that the application of adapalene after CP remission of acne treatment is an effective acne treatment method to maintain and further improve treatment effect and adherence in patients wishing to completely cure acne earlier. 


\section{CONFLICT OF INTEREST}

Adapalene and placebo were given by Galderma R\&D, France.

\section{ACKNOWLEDGEMENTS} Tokyo.

Funding for this study was provided by Galderma $R \& D$,

\section{REFERENCES}

[1] Arnold Odom J. William LAndrew's Diseases of the skin Clinical Dermatology. $8^{\text {th }}$ ed. Philadelphia: Harcourt Brace Jovanovich, Inc. 1990; pp. 250-7.

[2] Miyachi Y, Hayashi N, Furukawa F, et al. Acne management in Japan: study of patient adherence. Dermatology 2011;223(2): 17481 .

[3] Shimizu H. Shimizu's Textbook of Dermatology. Tokyo: Nakayama Ltd 2007; pp. 316-9.

[4] Harold J. Chemical Peeling and Resurfacing. St.Louis: Mosby Inc 1997; pp. 94-100.

[5] Yamamoto Y, Funasaka Y, Matsunaga K, et al. Guidelines for chemical peeling in Japan ( ${ }^{\text {rd }}$ edition). J Dermatol 2011; 38: 1-5.

[6] Hayashi N, Akamatsu H, Iwatsuki K, et al. The guideline for the treatment of acne vulgaris. Jpn J Dermatol 2004; 14: 195-202.

[7] Hayashi N, Akamatsu H, Kawashima M, et al. Establishment of grading criteria for acne severity. J Dermatol 2008; 35(5): 255-60.

[8] Michel S, Jomard A, Demarchez M. Pharamacology of adapalene. Br J Dermatol 1998; 139: 3-7
[9] Kawashima M, Harada S, Christian Loesche, et al. Adapalene gel $0.1 \%$ is effective and safe for Japanese patients with acne vulgaris: A ranomized, multicenter, investigator-blinded, controlled study. J Dermatol Sci 2008; 49(3): 241-8.

[10] Hayashi N, Kawashima M. The usefulness of chemical peeling with $30 \%$ glycolic acid (pH1.5) for acne vulgaris. Jpn J Clinic Dermatol 2003; 57: 1213-6 (in Japanese).

[11] Kishioka A, Yamamoto Y, Miyazaki T, et al. Clinical evaluation of chemical peeling with glycolic acid for acne. Aesthet Dermatol 2004; 14: 195-202.

[12] Hayashi N, Higaki Y, Kawamoto K, et al. A crosssectional analysis of quality of life in Japanese acne patients using the Japanese version of Skinde-16. J Dermatol 2004; 31(12): 971-6.

[13] Golden V, Clark SM, Cunliffe WJ. Post-adolescent-acne : a review of clinical features. Br J Dermatol 1997; 136(1): 66-70.

[14] Chiristina W, Layton AM. Persistent acne in woman. Am J Clin Dermatol 2006; 7(5): 281-90.

[15] George R, Clarke S, Thompson EB, et al. Hormonal therapy for acne. Semin Cutan Med Surg 2008; 27(3): 188-96.

[16] Watanabe R, Ito $M$. The structure and functional of sebaceous glands Aesthet Dermatol 2009; 19: 155-9 (abstract in English).

[17] Lee SH, Huh CH, Park KC, Youn SW.Effects of repetitive superficial chemical peels on facial sebum secretion in acne patients. J Eur Acad Dermatol Venereol 2006; 20: 964-8.

[18] Yonei N, Yamamoto Y, Kaminaka C, et al. Effect of chemical peeling with Keisei jorbi GA gel in treatment of acne vugaris, lichen pilaris, and postinflammatory pigmentation in aopic dermatitis. Aesthet Dermatol 2002; 12: 103-8 (abstract in English).

Received: June 24, 2013

Revised: August 5, 2013

Accepted: August 6, 2013

(C) Uede et al.; Licensee Bentham Open.

This is an open access article licensed under the terms of the Creative Commons Attribution Non-Commercial License (http://creativecommons.org/licenses/ by-nc/3.0/) which permits unrestricted, non-commercial use, distribution and reproduction in any medium, provided the work is properly cited. 